\title{
DESIGN OF AN INDIGENISED ELECTRONIC LOCK-IN AMPLIFIER
}

\author{
UMESH KUMAR* \\ Deptt. of Electrical Engineering, IIT, New Delhi-110016, India
}

(Received 7 April 2000; In final form 25 April 2000)

\begin{abstract}
An indigenised lock-in amplifier is designed that enables the accurate measurement of signals contaminated by broad-band noise, power-line pick-up, frequency drift, or other sources of interference. It does this by means of an extremely narrow band detector which has the centre of its passband locked to the frequency of the signal to be measured. Large improvements in signal-to-noise ratio are achieved.
\end{abstract}

Keywords: Lock-in; Amplifier; Narrow band detector; Accurate signal measurement

\section{INTRODUCTION}

The lock-in Amplifier is analogous to a synchronous demodulator or a phase sensitive detector. It is a specialized a.c. voltmeter that uses synchronous demodulation to measure signal strength or phase even under severe noise condition viz. where noise to signal ratio approaches $130 \mathrm{~dB}$. The instrument can be used wherever the signal of interest can be synchronised with or derived from a particular reference signal.

The output of a lock-in amplifier is a phase sensitive d.c. voltage proportional to the signal. It can be divided into four main sections: A signal channel; Reference Channel; Mixer (Phase sensitive detector) and a d.c. amplifier with a low-pass filter as shown in Figure 1.

*e-mail: umesh@ee.iitd.ernet.in 


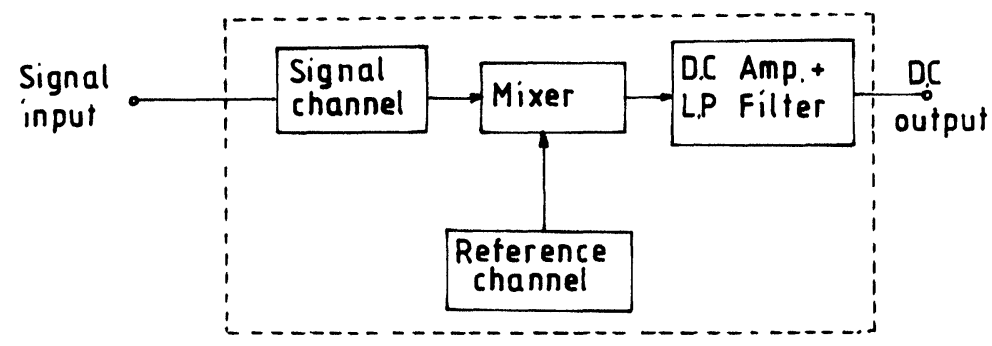

FIGURE 1

\section{DESCRIPTION OF THE VARIOUS PARTS}

In the signal channel, the input signal and noise is conditioned by the low-noise pre-amplifiers and post-amplifier with a filter sandwiched in between. The purpose of this filter is to reduce the possibility that the mixer will overload during severe noise conditions.

The reference channel transforms the externally applied reference to a suitable square wave at reference frequency to drive or switch the mixer. The lock-in output is independent of the referenced amplitude. However, the output does depend on the phase difference between the signal and the reference.

The Mixer is the main part of the lock-in amplifier. It is like an electronic reversing switch whose sense or position is determined by the square drive polarity of the reference channel. The signal channel output is commutated at the reference frequency. The signal channel output consists of two equal amplitude sinusoids, one in and one out of phase with the reference.

\section{DESCRIPTION OF OPERATION}

During the first half cycle, the reference drive is positive and the switch in Figure 2 is in position A. The mixer output is a positive half-wave sinusoid during this interval.

During the second half wave cycle, the switch is in position B and the mixer output is still positive. Therefore, when the reference and 

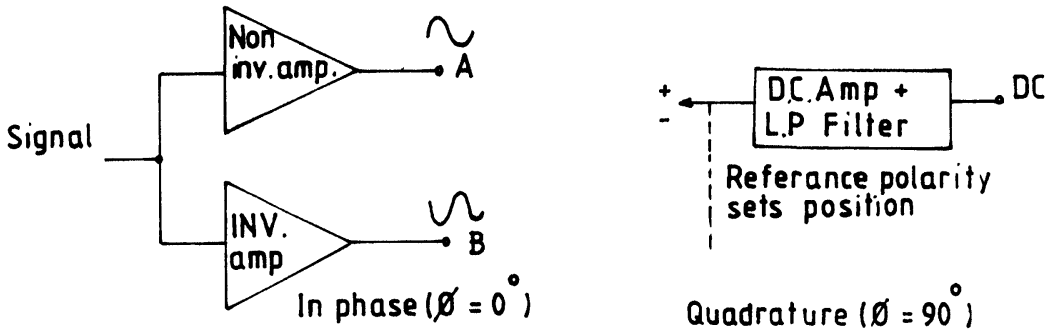

Quadrature $\left(\varnothing=90^{\circ}\right)$
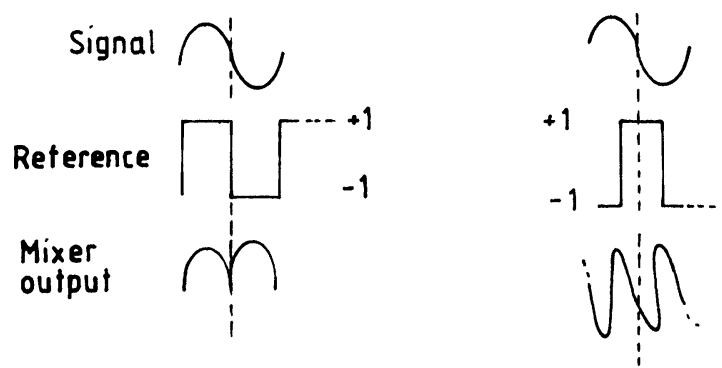

FIGURE 2

sinusoidal signals are in phase at the mixer input terminals, the mixer output is a full-wave rectified sinusoid whose fundamental is twice the reference frequency and whose d.c. component is proportional to the signal of interest. When the reference drive and the signal are in quadrature, that is their phase difference is 90 , there is no d.c. component.

Where the reference is not in phase with the signal, the mixer output will not contribute any d.c. component. This is what happens to any kind of noise present in the signal.

In the mathematical analysis of the demodulation process, the mixer output $m(t)$ can be thought of as a product of the signal channel output $s(t)$ and a \pm 1 Volt peak to peak square wave. With the reference represented by a Fourier series, $m(+)$ can be written as:

$$
m(t)=\sum_{n=0}^{\infty} s(t)\left\{\frac{4}{(2 n+1)} \sin \left[(2 n+1) \omega t+\phi_{n}\right]\right\}
$$




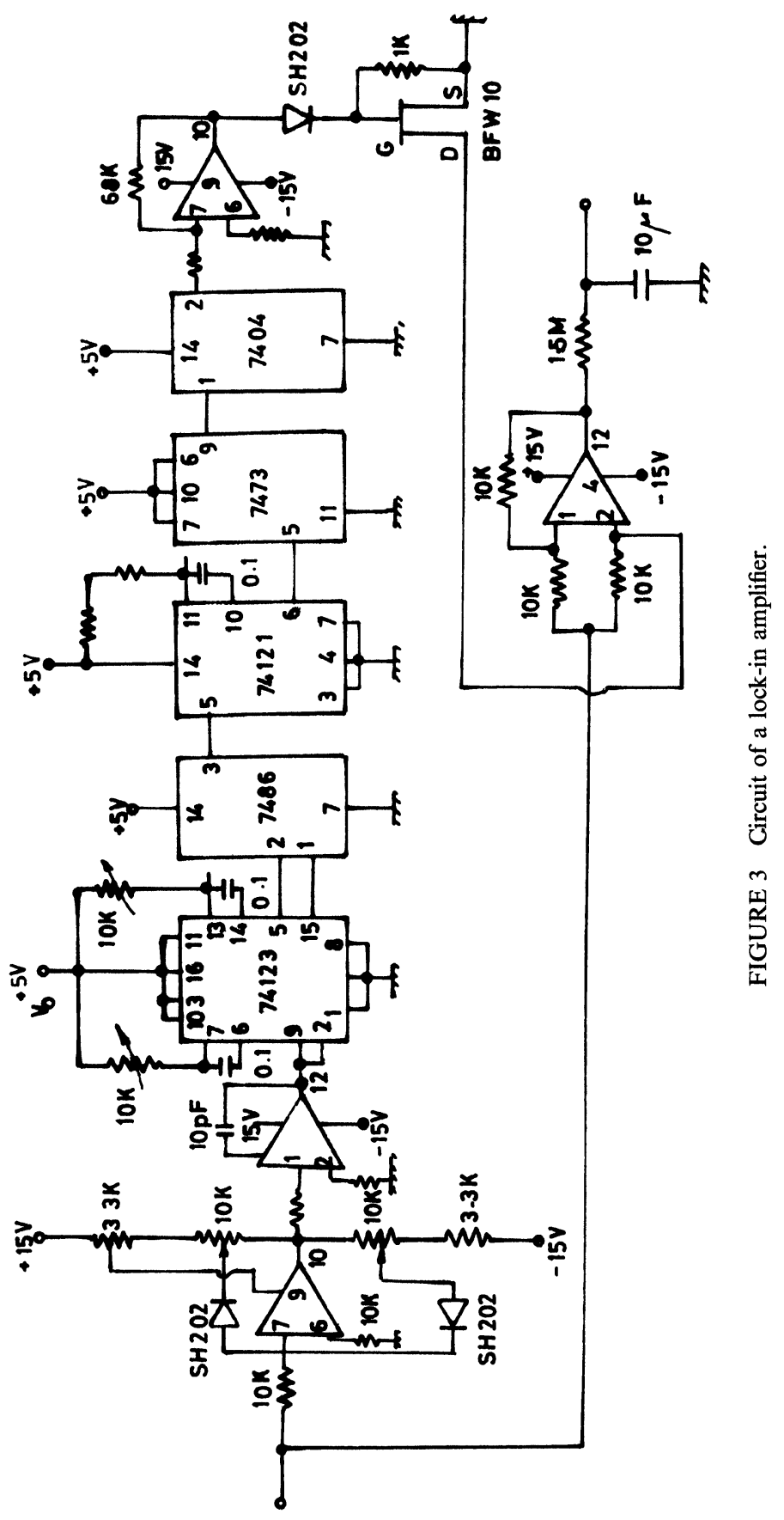


If $s(t)$ has a frequency component in common with any of the reference components, there will be a phase sensitive d.c. component.

Now synchronous components share no common frequencies with the reference and will not contribute d.c. components to the mixer output. When the mixer output is passed through a low pass filter to

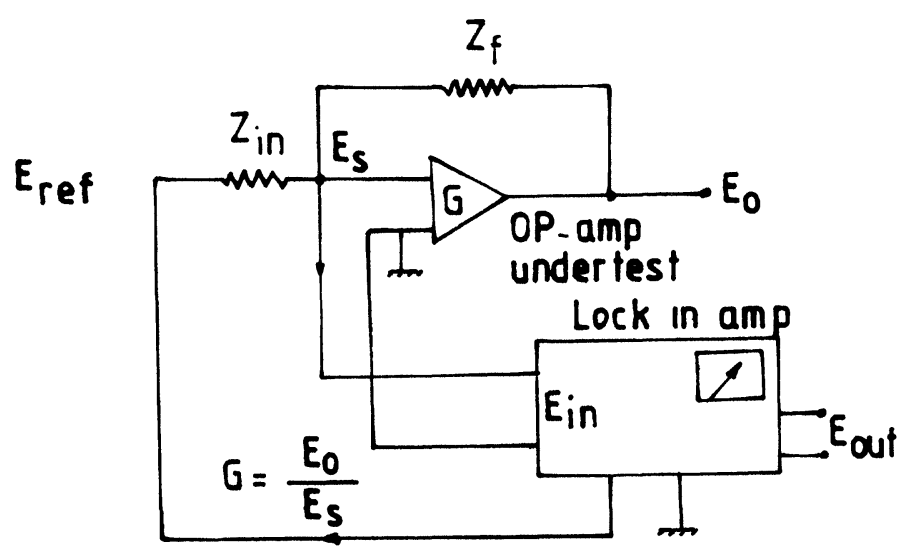

(a)

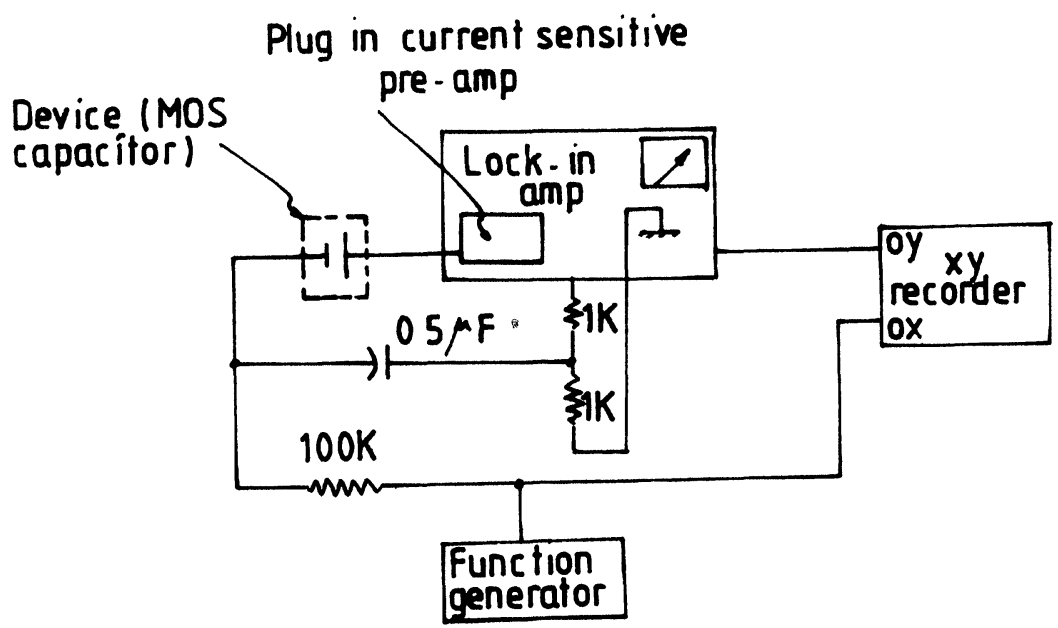

(b)

FIGURE 4 


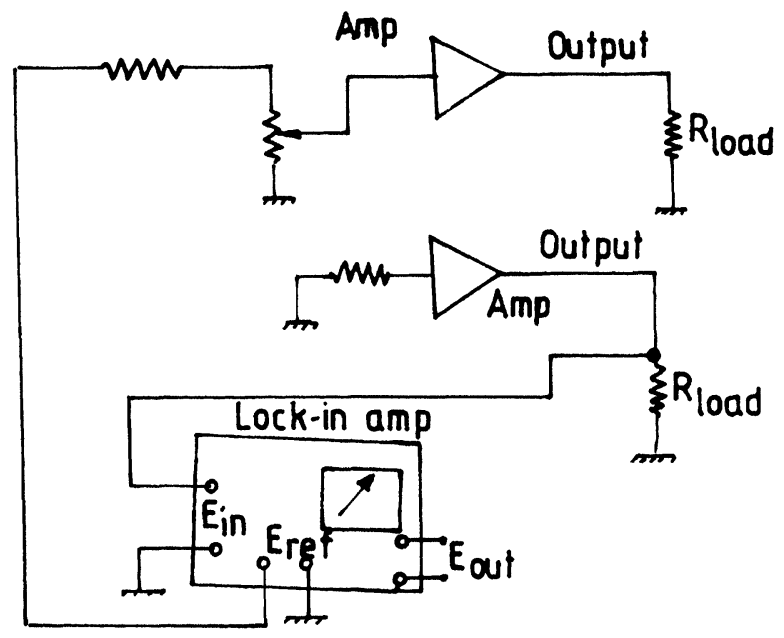

(c)

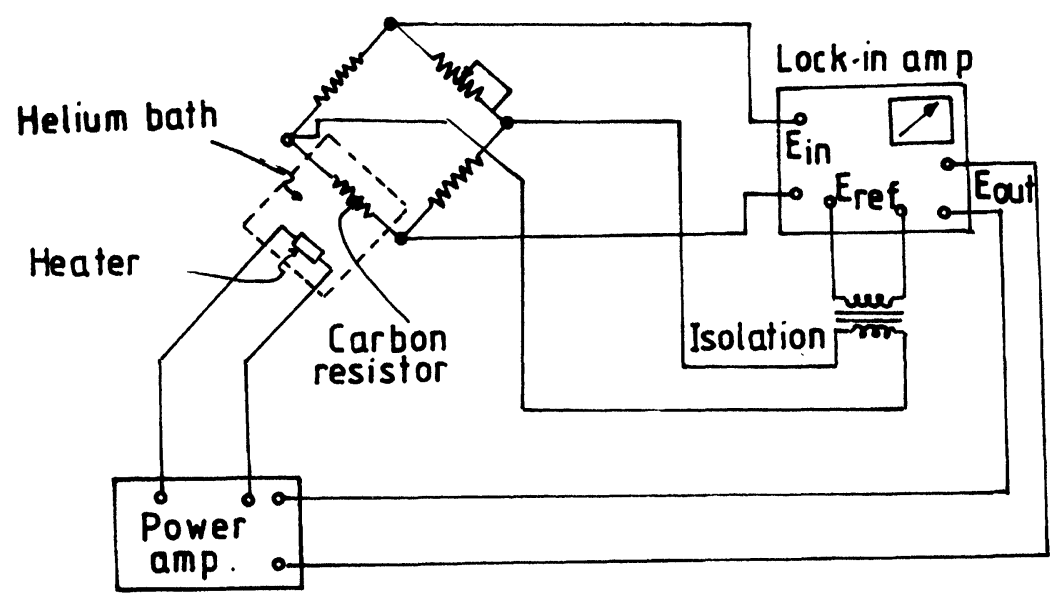

(d)

FIGURE 4 (Continued).

remove the a.c. fluctuations, the signal to noise ratio is enhanced in proportion to the square root of the filter time constant. Since the equivalent noise band width is determined by the low-pass filter and not the signal channel filter, band width is narrow. Figure 3 shows the actual indigenised circuit of a lock-in amplifier practically built and tested in the laboratory. 


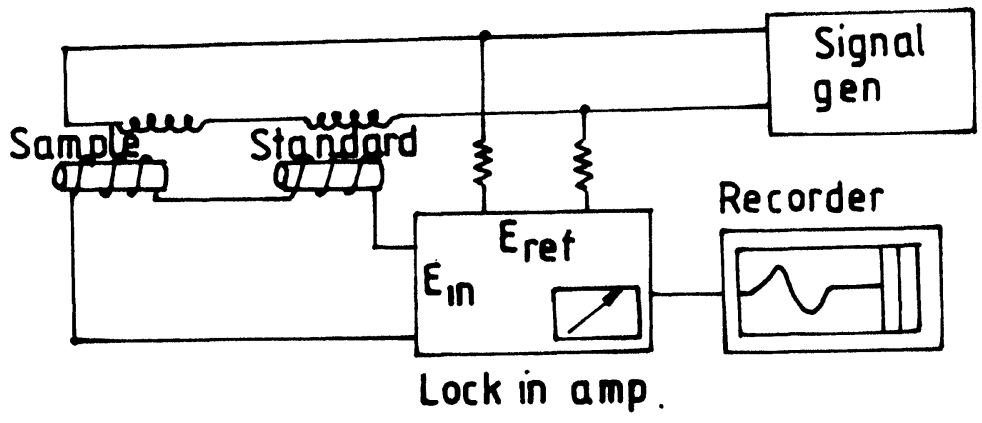

(e)

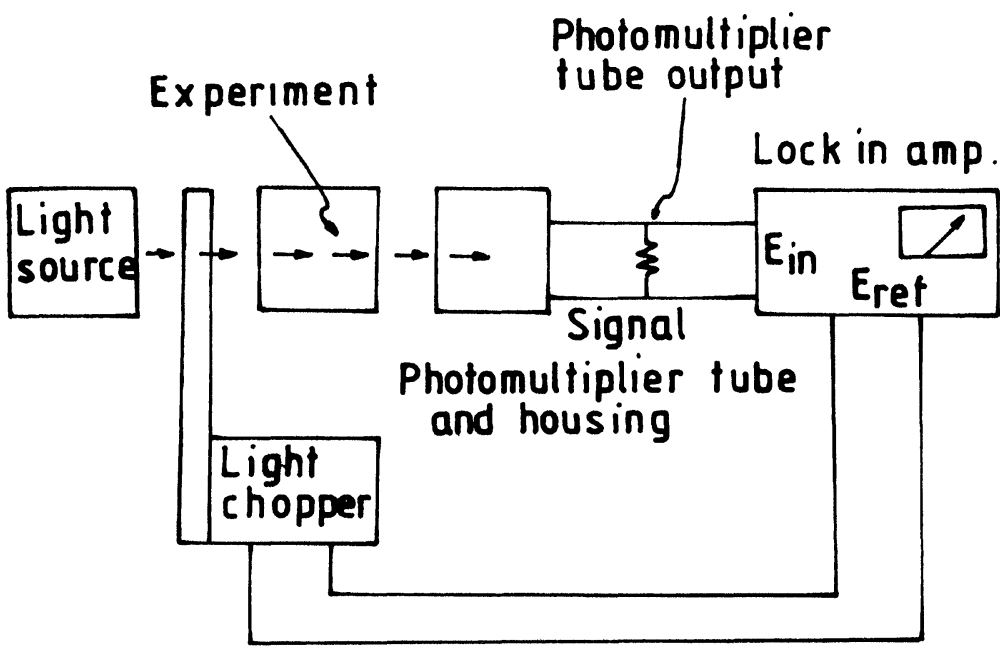

(f)

FIGURE 4 (Continued).

\section{APPLICATIONS}

Some typical applications of the lock-in amplifier are described below:

(a) To measure the open loop-gain of an operational amplifier (Fig. 4a).

(b) To determine the $C-V$ (capacitance-voltage) characteristics of a MOS Transistor (Fig. 4b). 
(c) To measure the amplifier cross talk (Fig. 4c).

(d) As a temperature controller (Fig. 4d).

(e) As an Eddy-current tester (Fig. 4e).

(f) Also as a photometric instrument (Fig. 4f).

\section{References}

[1] Letzter, S. G., "Explore the lock-in Amplifier". Electronic Design, 22, 104-109, 11 October, 1974.

[2] Macken, W. J., "Synchronous and Non-synchronous Rectification with op-amp", Electronic Engineering, 45, 18-19, March, 1973.

[3] Williams, D. R. and Lum, W. T. (1974). "Lock-in amplifier uses a single IC", Analog Dialogue, 8(1), $18-19$. 

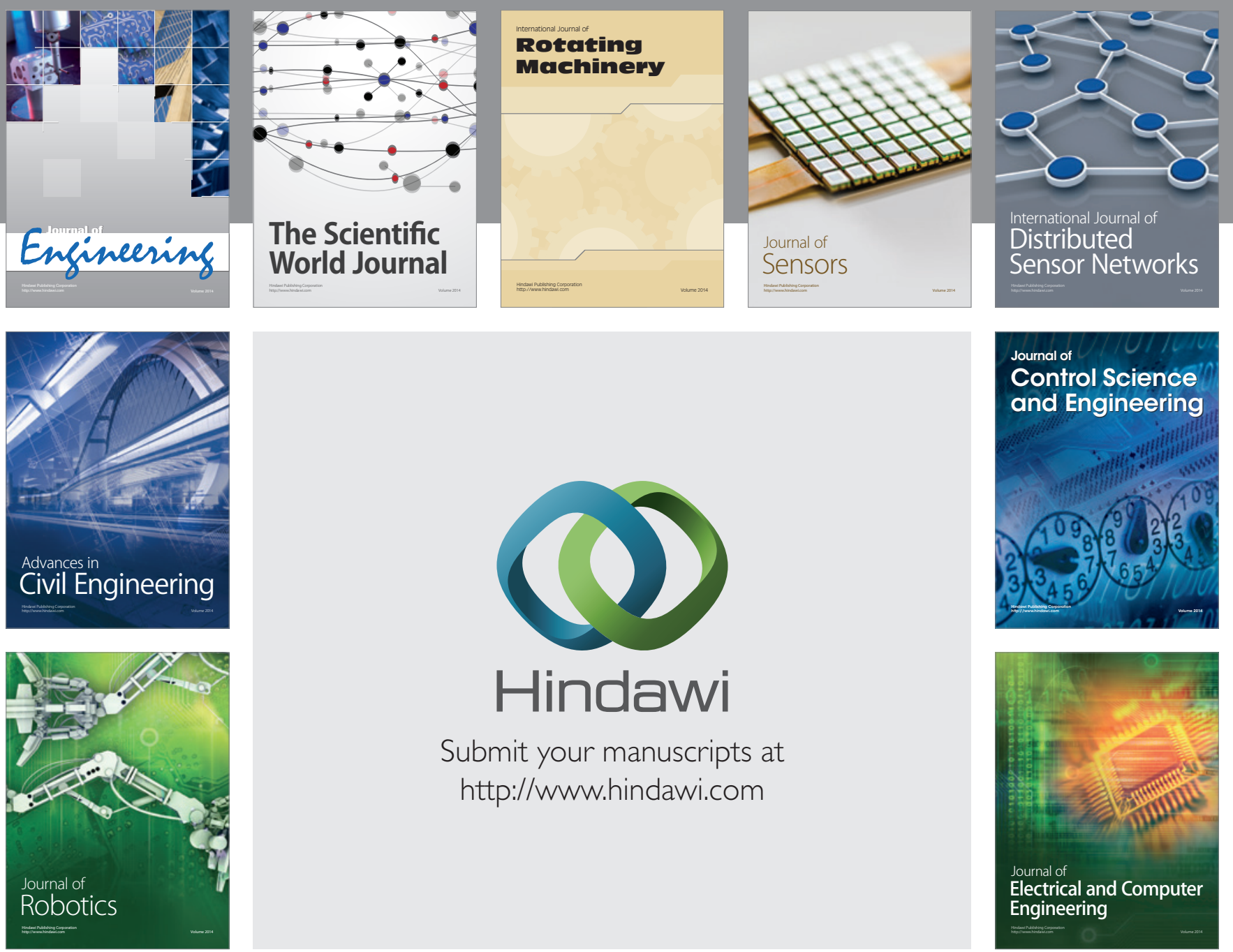

Submit your manuscripts at

http://www.hindawi.com
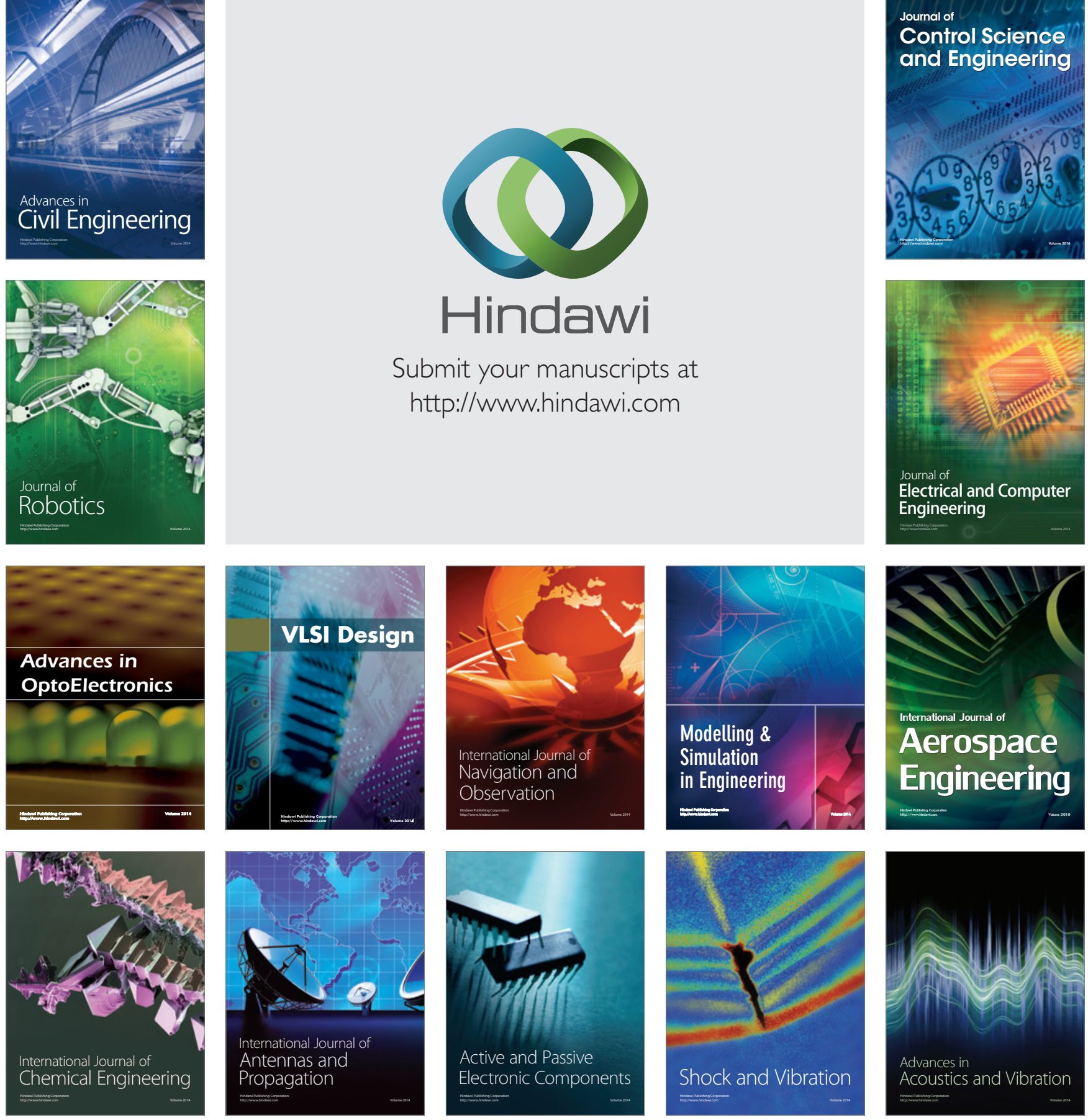